\title{
Elevación sinusal en pacientes con atrofia alveolar en el tratamiento de Implantología.
}

\author{
Sinus elevation in patients with alveolar atrophy in implantology \\ treatment.
}

Dania Rodríguez Herrera. ${ }^{1}$, María Teresa Lima Reyna. ${ }^{2}$ \& Rogelio Pérez Iglesias. ${ }^{3}$

\begin{abstract}
:
Resumen.

Introduction. Sinus elevation is a Introducción. La elevación sinusal es un predictable surgical procedure performed in order to vertically increase the amount of bone in the posterior region of the maxilla for implant-supported prosthetic rehabilitation. Objective: Characterize patients with alveolar atrophy in the oral implantology treatment of the posterior maxilla after elevation of the floor of the maxillary sinus. Methodology: An observational, descriptive and crosssectional investigation was carried out in the Maxillofacial Surgery service, procedimiento quirúrgico predecible que se realiza con la finalidad de aumentar verticalmente la cantidad de hueso en la región posterior del maxilar para la rehabilitación protésica implantosoportada. Objetivo: Caracterizar los pacientes con atrofia alveolar en el tratamiento de Implantología oral del maxilar posterior previa elevación del piso del seno maxilar. Metodología: Se realizó una investigación observacional, Hospital "Faustino Pérez" in Matanzas, descriptiva y transversal en el servicio de Cirugía Máxilo Facial, Hospital

\footnotetext{
${ }^{1}$ Hospital Universitario Clínico-Quirúrgico Faustino Pérez, Matanzas, Cuba, daniarodriguez890613@gmail.com iD https://orcid.org/0000-0002-6114-3932

${ }^{2}$ Hospital Universitario Clínico-Quirúrgico Faustino Pérez, Matanzas, Cuba, maytelimareina@gmail.com (iD https://orcid.org/0000-0002-7406-1186

${ }^{3}$ Universidad de Ciencias Médicas de Matanzas, Matanzas, Cuba. rogelioeduardopereziglesias@ gmail.com (iD) https://orcid.org/0000-0002-8933-4124
} 
between October 2017-September 2019.

The universe was made up of all the patients with posterior alveolar atrophy; a sample of 38 patients was taken.

Results: $47 \%$ of the patients corresponded to the age range 41-50 years, the female sex $82 \%$. 86 implants were placed, $69 \%$ in the premolar area, $31 \%$ in the molar area. $47 \%$ of the residual ridges had crestal height less than $5 \mathrm{~mm}$. There were a total of 51 sinus elevations, $71 \%$ were with the lateral window technique, $29 \%$ were transcrestal. $24 \%$ of the lateral window approach with crestal height less than $5 \mathrm{~mm}, 100 \%$ transcrestal between 8$10 \mathrm{~mm}$. 25 surgeries were unilateral and 13 bilateral. $17 \%$ of the implants were placed simultaneously, $83 \%$ delayed. $9.8 \%$ complications were recorded. Conclusions: Sinus elevation predominated in ages 41-50 years and in females. The most related teeth were the premolars. More than half of the patients with great bone deficit. A lateral window was made in cases with crestal height less than $8 \mathrm{~mm}$ with delayed implant placement. The transcrestal technique was performed from $8-10 \mathrm{~mm}$ with simultaneous implant placement. The most used technique was the lateral and unilateral localization window. The most frequent complication was perforation of Schneider's membrane in the lateral window technique.

Key Words: Maxillary sinus elevation, alveolar atrophy, dental implants.
"Faustino Pérez" de Matanzas, entre octubre 2017- septiembre 2019. El universo lo constituyó la totalidad de los pacientes con atrofia alveolar posterior; se tomó una muestra de 38 pacientes. Resultados: El $47 \%$ de los pacientes correspondió al rango etario 41-50 años, el sexo femenino el $82 \%$. Se colocaron 86 implantes, el $69 \%$ en zona de premolares, $31 \%$ en molares. El $47 \%$ de los rebordes residuales tenían altura crestal menor de $5 \mathrm{~mm}$. Hubo un total de 51 elevaciones sinusales, el $71 \%$ fue con la técnica ventana lateral, 29\% transcrestal. El 24\% del abordaje ventana lateral con altura crestal menor de $5 \mathrm{~mm}, 100 \%$ transcrestal entre $8-10 \mathrm{~mm}$. 25 cirugías fueron unilaterales y 13 bilaterales. El $17 \%$ de los implantes se colocaron simultáneos, $83 \%$ diferidos. Se registró $9.8 \%$ de complicaciones. Conclusiones: La elevación sinusal predominó en edades de 41-50 años y en el sexo femenino. Los dientes más relacionados fueron los premolares. Más de la mitad de los pacientes con gran déficit óseo. Se realizó ventana lateral en los casos con altura crestal menor de $8 \mathrm{~mm}$ con la colocación diferida del implante. La técnica transcrestal se realizó a partir de $8-10 \mathrm{~mm}$ con la colocación simultánea del implante. La técnica más usada fue la ventana lateral y de localización unilateral. La complicación más frecuente fue la perforación de la membrana de Schneider en la técnica ventana lateral.

Palabras Claves: Elevación de seno maxilar, atrofia alveolar, implantes dentales. 


\section{Introducción:}

La colocación de implantes en la zona posterior maxilar es actualmente un desafío en la rehabilitación fija implanto-soportada. Con la pérdida de las piezas dentales, los estímulos que mantienen el hueso alveolar desaparecen causando un proceso degenerativo que provoca el estrechamiento de la anchura de la cresta ósea, la disminución del trabeculado y de la altura, esta atrofia se acompaña de otras consecuencias, como disminución de tejidos blandos, alteración de la relación máxilo-mandibular, cambios faciales y de apariencia que afectan el pronóstico de esta zona como sitio receptor para colocación de implantes. Otros factores están también directamente relacionados, tales como, neumatización del seno, morfología inadecuada, calidad ósea de la región que es mayormente medular y la edad, que es inversamente proporcional a la densidad ósea. (Nasser Nasser K. , 2020).

Numerosos tratamientos han emergido en los últimos cincuenta años para corregir parcialmente esta dificultad, hasta que en los años ochenta surge la elevación del piso de seno maxilar como una alternativa quirúrgica válida que se ha ido afianzando en las últimas décadas como la más predecible. (Alsabbagh, Alsabbagh, Nahas, \& Rajih, 2017) (Deporter, Todescan, \& Caudry, 2017)

La elevación del seno maxilar es un procedimiento quirúrgico que sirve para incrementar la dimensión vertical del hueso en las porciones posterolaterales de los maxilares para posibilitar la inserción de implantes osteointegrados. (Avilés Echeverría, Hermida Salazar, \& Pineda Álvarez, 2020)

Las técnicas quirúrgicas de elevación del piso de seno maxilar se han clasificado en dos grandes grupos. Las de abordaje externo que requieren de una ventana ósea lateral para llegar al seno maxilar introducida en 1977 por Tatum y publicada en 1980 por Boyne y James. (Boyne \& James, 1980); y las de abordaje interno que llegan al seno maxilar a través del reborde óseo residual que es un procedimiento quirúrgico menos invasivo introducido por Summers en 1994 mediante el uso de osteótomos. (Summers, 1994)

La elevación lateral o directa del suelo del seno maxilar es una técnica quirúrgica más compleja que permite abordar esta estructura anatómica de una forma más amplia mediante una apertura o antrotomía vestibular y el despegamiento cuidadoso de la mucosa sinusal lo que permite desplazar hacia arriba el fragmento óseo, visualizando directamente la cavidad, la inserción de los implantes y rellenar, así mismo, con hueso autólogo y/o biomateriales ese espacio. (Nasser Nasser K. , 2020)

La técnica transalveolar o indirecta es menos invasiva y consiste en un abordaje en la cresta alveolar con un fresado controlado y la perforación del suelo antral, respetando la membrana sinusal y utilizando un instrumental adecuado (ej. osteótomos) con una suave presión e introduciendo y rellenando o no ese espacio neoformado con hueso y/o biomateriales. (Nasser Nasser K. , 2020)

En ambos grupos desde un punto de vista implantológico, existen dos alternativas para la inserción de los implantes. Una técnica supone la inserción de los implantes de forma 
simultánea a la elevación sinusal, en la misma sesión operatoria. Es decir, se realiza la elevación de la membrana sinusal por la vía lateral o transcrestal, simultáneamente se insertan los implantes por la vía alveolar y después del relleno del seno con biomaterial elegido. La segunda opción supone la realización de la elevación sinusal directa, y después de un periodo de tiempo de espera o cicatrización no menor de 6-8 meses se realiza la inserción diferida de los implantes. (Melej, Tirreau, \& Valdés, 2016)

La elección de la vía de abordaje y la colocación simultánea o diferida del implante depende de las distintas situaciones anatómicas y las diferentes topografías del seno respecto al reborde maxilar. En 1984 Carl Misch propuso una clasificación basada en la altura ósea residual en la que describe cuatro tipos de reborde residual cada uno con su opción terapéutica. (Misch, 1993)

Buco-palatino describió dos tipos:

- Tipo (A): 5 mm o más.

- $\operatorname{Tipo}(\mathrm{B}): 2.5$ a $5 \mathrm{~mm}$.

Vertical:

- Grado I. El hueso maxilar remanente es mayor de $10 \mathrm{~mm}$. Tratamiento: inserción de implantes de forma convencional sin elevación de seno.

- Grado II. El hueso maxilar remanente entre 8 y $10 \mathrm{~mm}$. Tratamiento: a) Colocación de implantes de diámetro ancho y longitud adecuada, sin romper la cortical sinusal. b) Colocación de implantes con elevación atraumática o pasiva del seno maxilar (empleando osteótomos).

- Grado III. La altura ósea residual está comprendida entre 5 y $8 \mathrm{~mm}$. Tratamiento: inserción de implantes con elevación traumática activa o con abordaje lateral del seno maxilar. En este caso no puede usarse la técnica anterior (elevación atraumática o pasiva del seno maxilar), ya que la mucosa sinusal no admite elongarse más de $5 \mathrm{~mm}$ sin perforarse, por lo que hay que abordar el seno con el método de Tatum

- Grado IV. El hueso maxilar remanente es menor de $5 \mathrm{~mm}$. Tratamiento: elevación del seno maxilar por vía lateral e inserción diferida de los implantes en un segundo tiempo quirúrgico, después de la maduración del injerto antral.

El indica la antroplastía en los casos en que existe una altura crestal de 5 a $8 \mathrm{~mm}$, lo que clasifica como SA3 y SA4.

En estos procedimientos se han utilizado injertos óseos de diferentes orígenes: hueso autólogo, aloinjertos, xenoinjertos y materiales aloplásticos. Todos ellos han mostrados resultados aceptables. (C.D Aguirre Valencia, 2019) (Lambert, y otros, 2017)

Debido a que, en los últimos años, se ha proyectado una gran cantidad de factores que influencian la predictibilidad de la elevación del piso de seno maxilar; ello lo convierte en un procedimiento en constante estudio. Sin embargo, la evidencia en la provincia Matanzas se encuentra aislada, en la minuciosa búsqueda bibliográfica realizada para este 
estudio, no se encontró ninguna investigación que se ocupe de la observación del tema dirigidos al profesional de estomatología. Se vio entonces la necesidad de revisar y reportar la evidencia disponible acerca de las implicaciones anatómicas, clínicas y radiográficas del procedimiento de elevación sinusal para la colocación de implantes.

El objetivo de la investigación fue caracterizar los pacientes con atrofia alveolar en el tratamiento de Implantología oral previa elevación del piso seno maxilar.

\section{Metodología:}

Se realizó una investigación observacional descriptiva transversal en el servicio de Cirugía Máxilo Facial del Hospital Universitario "Faustino Pérez "del municipio Matanzas; en el período comprendido entre octubre del 2017 a septiembre del año 2019.

El universo estuvo constituido por la totalidad de los pacientes con atrofia alveolar que fueron 38 y a los que se les realizó 51 elevaciones del piso de seno maxilar.

Antes del tratamiento se examinó cada caso mediante radiografías simples (ortopantomografía, proyección de Waters y Rx periapical) con el fin de analizar la situación ósea en cuanto a sus dimensiones en anchura y altura, la cual fue medida mediante un pie de rey, registrándose en milímetros la distancia del reborde alveolar. Se incluyeron aquellos pacientes con ausencia de volumen óseo necesario para insertar implantes; señalando una serie de requisitos básicos:

- Altura del reborde alveolar residual menor de $10 \mathrm{~mm}$.

- Espesor del reborde alveolar residual menor de $4 \mathrm{~mm}$.

- Ausencia de patología sinusal previa.

- Ausencia de limitaciones anatómicas.

Se excluyeron los pacientes con:

- Presencia de patología sinusal que pudiera interferir con el tratamiento.

- Inadecuada dimensión transversa del seno.

- Inadecuado o gran espacio entre arcos. Inapropiada relación intermaxilar.

- Patología periapical asociada. Enfermedad periodontal activa y sin tratamiento.

- Enfermos terminales.

- Factores que disminuyan la supervivencia del injerto.

- Factores que contraindiquen la inserción de implantes. Embarazo. Diabetes Mellitus no controlada.

Siguiendo la clasificación de Misch (Misch, 1993) según el espacio disponible del reborde residual alveolar se seleccionó la técnica quirúrgica a emplear.

En todos los casos, se utilizó como biomaterial de relleno betafosfatotricálcico y membrana de colágeno. Los implantes insertados fueron Miss Seven.

Después de la cirugía, la cual se realizó con anestesia local los pacientes comenzaron un régimen con antibiótico preventivo (amoxicilina + metronidazol) durante 10 días. Así 
mismo, todos los pacientes fueron tratados durante una semana con ibuprofeno, gotas nasales e inhalaciones, según protocolo de tratamiento de la sinusitis postoperatoria.

A la semana se retiraron las suturas y a todos los pacientes se les recomendó el enjuague diario con clorhexidina durante los primeros 30 días.

A los 6 meses en los implantes insertados de forma simultánea y a los 8 meses en los insertados de forma diferida a la elevación sinusal, se realizó la carga funcional de los implantes mediante la colocación de las correspondientes prótesis fijas implantosoportadas ya que es el tiempo mínimo necesario para la consolidación del material injertado.

Se tuvo en cuenta los principios bióticos, implícitos en todo estudio realizado con seres humanos. Los pacientes fueron informados de la técnica quirúrgica de elevación sinusal con sus posibles complicaciones y fracasos. Así mismo, se explicó el protocolo posterior del tratamiento con implantes, de los aspectos quirúrgicos y prostodóncicos, temporalización del tratamiento y seguimiento, así como de la posibilidad de la existencia de complicaciones y pérdida de implantes. En la información interpersonal no se empleó lenguaje técnico, sino sencillo y comprensible para el sujeto. La información relacionada con su identidad fue tratada de manera confidencial. Los investigadores no coaccionaron, ni influyeron para participar o mantenerse en el estudio, considerando este la máxima expresión del principio de autonomía.

La fuente de información que se empleó fue la historia clínica de cada paciente. Para el análisis estadístico de las variables: sociodemográficas, relaciones anatómicas dentales, altura crestal, técnicas quirúrgicas, localización, colocación de implantes y complicaciones se creó una base de datos mediante el software SPSS, versión 20. Se utilizó el porciento como medida de resumen realizando distribuciones de frecuencia a todas las variables en estudio. Se presentaron los resultados en tablas de distribución de frecuencias que fueron analizadas y discutidas de acuerdo a los objetivos propuestos para emitir las conclusiones en contraste y comparación con los resultados encontrados en la literatura científica.

\section{Resultados:}

La distribución de pacientes con elevación del piso del seno maxilar según grupo de edad y sexo (tabla 1) reflejó que el grupo etario que predominó fue el comprendido entre 4150 años (47\%). Con relación al sexo el mayor porciento lo constituyó el femenino con un $82 \%$ del total. Los resultados obtenidos se deben a que las mujeres son más preocupadas que los hombres por su estética y salud bucal, además la elevación del piso del seno maxilar es un tratamiento pre-implantológico que requiere de cuidados pre y postoperatorios para evitar complicaciones y los hombres manifiestan más el miedo por lo que la mayoría prefieren rehabilitarse protésicamente con los métodos convencionales. (Esposito, y otros, 2010), en un artículo sobre la efectividad de la elevación del piso del seno maxilar para la rehabilitación con implantes dentales obtuvo resultados similares a esta investigación, siendo el sexo femenino la mayor representación con un $72 \%$ y la edad media 49 años. 
Tabla 1: Distribución de los pacientes con elevación del piso del seno maxilar según grupo de edad y sexo. Hospital Faustino Pérez. Matanzas. Octubre 2017- diciembre 2019.

\begin{tabular}{llllllll}
\hline $\begin{array}{c}\text { Grupos } \\
\text { de } \\
\text { Edades }\end{array}$ & Masculino & & $\%$ & Femenino & $\%$ & Total & $\%$ \\
\cline { 2 - 8 } & 1 & 14 & 2 & 6 & 3 & 8 \\
$31-40$ & 3 & 43 & 5 & 16 & 8 & 21 \\
$41-50$ & 1 & 14 & 17 & 55 & 18 & 47 \\
$51-60$ & 1 & 14 & 6 & 19 & 7 & 18 \\
61 & 0 & 1 & 14 & 1 & 3 & 2 & 5 \\
más & 1 & 18 & 31 & 82 & 38 & 100 \\
Total & 7 & & & & & & \\
\hline
\end{tabular}

Fuente: Historias Clínicas de Implantología oral.

Cuando se profundiza en las relaciones anatómicas dentales con el seno maxilar (tabla 2); se observó que hubo un predominio de los premolares (69\%) sobre los molares (31\%) para un total de 86 implantes posteriores al tratamiento. El 61\% correspondió al segundo premolar. El primer molar alcanzó el 93\% coincidiendo que el grupo de edad de 41-50 años es la población más tratada con un $60 \%$. Solamente un $7 \%$ correspondió al segundo molar en el rango de 41-50 años. El estudio coincide con muchos investigadores (Cosci \& Luccioli, 2000), (Duan, y otros, 2017), (Herrmann, Lekholm, Holm, \& Kultje, 2005), (Jiménez, y otros, 2015), que plantean que las zonas más intervenidas son las relacionadas con el segundo premolar y el primer molar porque los ápices de estos dientes mantienen una estrecha relación con el suelo del seno maxilar, además el primer molar es el diente que más rápido se pierde al ser el primero en brotar y muchas veces desde edades tempranas, por lo que la reabsorción ósea y neumatización es mayor a este nivel y anatómicamente es el punto de mayor convexidad del piso sinusal.

Tabla 2: Distribución de pacientes según grupos de edades y relaciones anatómicas dentales con el seno maxilar.

\begin{tabular}{|c|c|c|c|c|c|c|c|c|}
\hline \multirow{3}{*}{ Grupos de Edades } & \multicolumn{8}{|c|}{$\begin{array}{l}\text { Total } \\
\mathrm{N}=86\end{array}$} \\
\hline & \multicolumn{4}{|c|}{$\begin{array}{l}\text { Pre-Molares } \\
\qquad n=59\end{array}$} & \multicolumn{4}{|c|}{$\begin{array}{c}\text { Molares } \\
n=27\end{array}$} \\
\hline & $1 \mathrm{er}$ & $\%$ & 2do & $\%$ & $1 e r$ & $\%$ & 2do & $\%$ \\
\hline $18-30$ & 1 & 4 & 3 & 8 & 1 & 4 & - & - \\
\hline $31-40$ & 5 & 22 & 6 & 17 & 2 & 8 & - & - \\
\hline $41-50$ & 9 & 39 & 16 & 44 & 15 & 60 & 2 & 100 \\
\hline $51-60$ & 5 & 22 & 7 & 19 & 4 & 16 & - & - \\
\hline
\end{tabular}




\begin{tabular}{lllllllll}
\hline 61 o más & 3 & 13 & 4 & 11 & 3 & 12 & - & - \\
Total & 23 & 39 & 36 & 61 & 25 & 93 & 2 & 7
\end{tabular}

Fuente: Historias Clínicas de Implantología oral.

De un total de 51 elevaciones del piso del seno maxilar en 38 pacientes; hubo un predominio de senos con gran déficit óseo donde la altura crestal fue menos de $5 \mathrm{~mm}$ (Tabla 3). Diversos estudios, (Avilés Echeverría, Hermida Salazar, \& Pineda Álvarez, 2020), (Cosci \& Luccioli, 2000), (Esposito, y otros, 2010) (Guzman Cruz \& Sauri Esquivel, 2017), (Shalu, Ramesh, \& Komal, 2018) evalúan los resultados de la elevación del piso del seno maxilar y la colocación de implantes según la altura crestal coincidiendo que casi el 50\% de los pacientes presentan altura crestal menor de $5 \mathrm{~mm}$. Como tendencia se observó que a medida que aumenta la edad el déficit óseo es mayor igual que los resultados reportados en esta investigación. (Avila-ortiz, Wang, Galindo-moreno, Misch, \& Rudek, 2012) señaló que la cantidad de hueso disponible no será el único factor a tener en cuenta, ya que el patrón de reabsorción y la calidad del hueso también van a condicionar el tratamiento implantológico a corto y largo plazo. Es criterio de los autores que un aumento sinusal es exitoso cuando el hueso disponible en altura, después de la intervención, asegura la inserción de implantes con un mínimo de $10 \mathrm{~mm}$ de longitud y un diámetro de $4 \mathrm{~mm}$, sin precisar la edad del paciente, lo cual fue demostrado en el presente estudio ya que todos los implantes insertados se corresponden con estas características macroscópicas.

Tabla 3: Clasificación de la pérdida de hueso en sentido vertical según grupo de edades.

\begin{tabular}{llllllll}
\hline \multirow{2}{*}{$\begin{array}{c}\text { Grupos de } \\
\text { Edades }\end{array}$} & $\begin{array}{c}\text { No de } \\
\text { Pacientes }\end{array}$ & \multicolumn{6}{c}{ Altura Crestal } \\
\cline { 5 - 8 } & & $\begin{array}{l}\text { Mayor } 10 \\
\mathrm{~mm}\end{array}$ & $\begin{array}{l}\mathrm{e} / \mathrm{8} \\
\mathrm{mm}\end{array}$ & $\begin{array}{l}-10 \\
\mathrm{e} / \mathrm{5}-8 \\
\mathrm{~mm}\end{array}$ & $\begin{array}{l}\text { Menor } \\
\mathrm{mm}\end{array}$ & $\mathbf{5}$ & Total \\
\hline $18-30$ & 3 & - & 1 & 2 & - & 3 \\
$31-40$ & 8 & - & 4 & 4 & 2 & 10 \\
$41-50$ & 18 & - & 6 & 6 & 13 & 25 \\
$51-60$ & 7 & - & 4 & - & 5 & 9 \\
61 o más & 2 & - & - & - & 4 & 4 \\
Total & 38 & - & 15 & 12 & 24 & 51 \\
$\%$ & & - & 29 & 24 & 47 & 100
\end{tabular}

Fuente: Historias Clínicas de Implantología oral.

La distribución de los pacientes según grupo de edades y técnica quirúrgica (Tabla 4) arrojó que el abordaje abierto con ventana lateral representó el $71 \%$ del total de las técnicas quirúrgicas y el 29\% restante correspondió al abordaje transcrestal. El $49 \%$ del total de las intervenciones realizadas correspondió al grupo de edades de 41 a 50 años; esto se debió en gran medida a que el mayor número de pacientes atendidos se correspondió a este rango de edad. En varios reportes revisados (Guzman Cruz \& Sauri 
Esquivel, 2017), (Jiménez, y otros, 2015), (Melej, Tirreau, \& Valdés, 2016), (Shalu, Ramesh, \& Komal, 2018), se observó que el $60 \%$ de las elevaciones sinusales con la técnica ventana lateral se realiza en pacientes con más de 40 años, dado a la atrofia alveolar progresiva propia de la edad.

Tabla 4: Distribución de pacientes según grupo de edad y técnica quirúrgica.

\begin{tabular}{|c|c|c|c|c|c|c|}
\hline \multirow{2}{*}{$\begin{array}{c}\text { Grupos } \\
\text { de } \\
\text { Edades }\end{array}$} & \multicolumn{6}{|c|}{ Técnica Quirúrgica } \\
\hline & Transcrestal. & $\%$ & Ventana Lateral & $\%$ & Total & $\%$ \\
\hline $18-30$ & 1 & 7 & 2 & 6 & 3 & 6 \\
\hline $31-40$ & 4 & 27 & 6 & 17 & 10 & 20 \\
\hline $41-50$ & 6 & 40 & 19 & 53 & 25 & 49 \\
\hline $51-60$ & 4 & 27 & 5 & 14 & 9 & 18 \\
\hline $\begin{array}{l}61 \\
\text { más }\end{array}$ & - & - & 4 & 11 & 4 & 8 \\
\hline Total & 15 & 29 & 36 & 71 & 51 & 100 \\
\hline
\end{tabular}

Fuente: Historias Clínicas de Implantología oral.

La relación de la técnica quirúrgica según la altura crestal (Tabla 5) mostró que del total de 51 elevaciones sinusales, el $67 \%$ de la ventana lateral tenían una altura crestal menor de $5 \mathrm{~mm}$ y el $33 \%$ entre 5-8 $\mathrm{mm}$. Se realizó la técnica transcrestal a la totalidad de los casos con altura crestal de 8-10 mm. Estos resultados coinciden con (Esposito, y otros, 2010) el cual realizó la elección de la técnica quirúrgica a expensas del volumen óseo para la colocación simultánea o diferida del implante, predominando la ventana lateral en el 65\% de los casos con altura crestal de $5 \mathrm{~mm}$ o menos según la clasificación de Misch. Existen estudios (Alsabbagh, Alsabbagh, Nahas, \& Rajih, 2017), (Bensaha \& Mjabber, 2016), en que comparan resultados de tratamientos con ambas técnicas quirúrgicas en diferentes pacientes que presentan una situación clínica similar donde cada enfoque clínico va a tener unas ventajas y unas desventajas: la técnica transcrestal es más conservadora que la convencional y se coloca el implante en el mismo acto quirúrgico pero la desventaja es que se realiza a ciegas.

La ventana lateral se puede colocar el implante simultáneo cuando se dispone de $5 \mathrm{~mm}$ o diferido cuando se desea recuperar grandes volúmenes de hueso, porque reduce la posibilidad de lesionar los senos maxilares durante la colocación de implantes, permite el crecimiento de hueso propio para restaurar el hueso perdido y permite la creación de un entorno adecuado para la colocación de implantes; pero en este caso el plan de tratamiento suele ser complejo y el tiempos de cicatrización y rehabilitación implanto protésica prolongados. (Herrmann, Lekholm, Holm, \& Kultje, 2005) refirió en su investigación que es difícil comparar el gran número de estudios sobre técnicas de elevación del seno maxilar en relación a la altura ósea disponible por la diferencia existente en cuanto a criterios de inclusión y exclusión, grado de reabsorción, fases de tratamiento, tipo de material de injerto y la elección del cirujano. Los autores coinciden con Herrmann porque, 
aunque teóricamente se han definido las indicaciones de ambos abordajes estos se han ampliado al desarrollarse nuevas tecnologías que facilitan o mejoran los procedimientos haciéndolos más versátiles.

Es importante aclarar que la investigación que se muestra no constituye un reporte comparativo, se centra en la valoración de la evolución de un grupo de pacientes a partir de la técnica empleada de acuerdo a la altura crestal y las características de cada paciente.

Tabla 5: Relación de técnica quirúrgica según altura crestal.

\begin{tabular}{|c|c|c|c|c|c|c|}
\hline \multirow{2}{*}{$\begin{array}{l}\text { Altura } \\
\text { Crestal }\end{array}$} & \multicolumn{6}{|c|}{ Técnica Quirúrgica } \\
\hline & Transcrestal & $\%$ & Ventana lateral & $\%$ & Total & $\%$ \\
\hline $\begin{array}{l}\text { Mayor } \\
10 \mathrm{~mm}\end{array}$ & - & - & - & - & - & - \\
\hline $\begin{array}{l}\mathrm{e} / 8- \\
10 \mathrm{~mm}\end{array}$ & 15 & 100 & - & - & 15 & 29 \\
\hline $\begin{array}{l}\mathrm{e} / 5-8 \\
\mathrm{~mm}\end{array}$ & - & - & 12 & 33 & 12 & 24 \\
\hline $\begin{array}{l}\text { Menor } \\
5 \mathrm{~mm}\end{array}$ & - & - & 24 & 67 & 24 & 47 \\
\hline Total & 15 & 29 & 36 & 71 & 51 & 100 \\
\hline
\end{tabular}

Fuente: Historias Clínicas de Implantología oral.

De acuerdo a la técnica quirúrgica y su localización en el maxilar (Tabla 6) se obtuvo que del total de 38 pacientes hubo un predominio de las cirugías unilaterales. En sentido general la técnica quirúrgica ventana lateral se realizó tanto en la localización unilateral como bilateral. Estudios revisados por el autor (Guzman Cruz \& Sauri Esquivel, 2017) reportan que los mayores por ciento de las elevaciones del piso del seno maxilar previo a la rehabilitación con implantes son de localización unilateral, clase II de Kennedy con un $80,5 \%$ y el $19.5 \%$ bilateral. Estos resultados coinciden con los obtenidos en este estudio como se describió anteriormente.

Tabla 6: Técnica quirúrgica según localización.

\begin{tabular}{lllllll}
\hline Técnica & \multicolumn{2}{l}{ Localización } & & & \\
\cline { 2 - 7 } Quirúrgica & Unilateral & $\%$ & Bilateral & $\%$ & Total & $\%$ \\
\hline Transcrestal. & 11 & 44 & - & - & 11 & 29 \\
$\begin{array}{l}\text { Ventana } \\
\text { Lateral }\end{array}$ & 14 & 56 & 9 & 69 & 23 & 61 \\
Combinadas & - & - & 4 & 31 & 4 & 10 \\
Total & 25 & 100 & 13 & 100 & 38 & 100 \\
\hline
\end{tabular}

Fuente: Historias Clínicas de Implantología oral. 
En el periodo se colocaron 15 implantes simultáneos a la técnica quirúrgica transcrestal con altura crestal de 8-10 mm ya que en esta situación clínica se consigue una buena estabilidad primaria de los implantes. Sin embargo, con el abordaje ventana lateral se colocaron 71 implantes diferidos porque no ofrece una estabilidad primaria aceptable, al haber un mayor déficit óseo (altura menor de $5 \mathrm{~mm}$ ) (Tabla 7); en estos casos se decidió esperar 6 meses para la inserción de implantes en una segunda fase.

Los autores consideran importante colocar los implantes diferidos en los pacientes con elevación sinusal traumática porque en estos casos hubo mayor atrofia alveolar con el inconveniente de que el tratamiento rehabilitador con la carga funcional del implante requiere el doble de tiempo y que el paciente es sometido a dos intervenciones quirúrgicas, pero vale aclarar que muchos autores (Alsabbagh, Alsabbagh, Nahas, \& Rajih, 2017), (Jiménez, y otros, 2015) plantean que esperar 6 meses es favorable porque es el tiempo mínimo necesario para la consolidación del material injertado asegurando una buena regeneración ósea y favorece una buena estabilidad primaria de los implantes. La reacción ósea después de la intervención suele ser muy favorable. El material injertado y el hueso neoformado parecen remodelarse adecuadamente como reacción a la carga funcional de los implantes.

(Bustillo, 2017) difiere de este criterio porque plantea que en un periodo de 6 meses el seno maxilar tiende a reneumatizarse, colocando los implantes simultáneos al abordaje ventana lateral. Estudios revisados (Herrmann, Lekholm, Holm, \& Kultje, 2005), (Jiménez, y otros, 2015) no constatan la reabsorción del biomaterial en este tiempo y los implantes colocados en una segunda fase fueron estables igual que los resultados de nuestra investigación. Por su parte, (Esposito, y otros, 2010) compara en su estudio la colocación simultánea o diferida del implante, concluyendo que ambas opciones fueron capaces de lograr las metas planeadas, sin diferencias estadísticas significativas. Sin embargo, uno de los implantes analizados no obtuvo la estabilidad primaria necesaria, sugiriendo que el riesgo es real, y que, en estos casos, en que se sospeche de antemano que no se va a lograr la estabilidad, sería mejor posponer la instalación del implante y realizar el procedimiento en 2 tiempos quirúrgicos.

(Nasser Nasser K. e., 2018) concluyó en su estudio que el procedimiento usando la vía crestal es menos invasivo, reduce el tiempo operatorio, y minimiza el discomfort postoperatorio, mostrando que esta técnica es segura para rebordes residuales con 5 o 6 $\mathrm{mm}$ de altura, déficit de anchura ósea de aproximadamente 1.5 a $2.5 \mathrm{~mm}$; y una baja densidad ósea. Con este tipo de procedimientos se obtiene hasta $13 \mathrm{~mm}$ en altura, conservando e incrementando la cantidad ósea. A pesar de los resultados de este autor en nuestra investigación optamos por realizar la técnica transcrestal a partir de $8 \mathrm{~mm}$ con el objetivo de ganar $5 \mathrm{~mm}$ evitando el hiperextensión de la membrana para evitar la perforación de la misma, la cual no tenemos control visual de la misma con esta elevación atraumática.

Los autores consideran que la cantidad de hueso cortical es un factor decisivo en la colocación de implantes simultáneos a la elevación del suelo sinusal; a pesar de esto en el mundo, la rehabilitación con implantes no solo se utiliza para mejorar la salud bucal de 
los pacientes, también se usa como negocio, resultando un tratamiento muy caro en el sector privado y muchos pacientes buscan opciones más rápidas de tratamiento, sin importar las características clínicas, lo que después constituye un fracaso y la tasa de supervivencia de los implantes es menor por las complicaciones del tratamiento. Debido a los pequeños tamaños de muestra, y a la amplia heterogeneidad en tiempos de instalación, tipos de implantes, altura ósea residual, número de implantes, biomateriales, etc., que fue evaluada por cada trabajo, son necesarios más estudios clínicos aleatorizados que evalúen parámetros estandarizados y con tamaño de muestras mayores para poder poseer información más concluyente.

Tabla 7: Colocación de Implantes según Técnicas Quirúrgicas.

\begin{tabular}{|c|c|c|c|c|c|c|}
\hline \multirow{3}{*}{$\begin{array}{l}\text { Técnica } \\
\text { Quirúrgica }\end{array}$} & \multicolumn{6}{|c|}{ Colocación de Implantes } \\
\hline & \multicolumn{2}{|c|}{ Simultáneos } & \multicolumn{2}{|c|}{ Diferidos } & \multicolumn{2}{|c|}{ Total } \\
\hline & No & $\%$ & No & $\%$ & No & $\%$ \\
\hline $\begin{array}{l}\text { Transcrestal } \\
n=15\end{array}$ & 15 & 100 & - & - & 15 & 17 \\
\hline $\begin{array}{l}\text { Ventana Lateral } \\
\mathrm{n}=36\end{array}$ & - & - & 71 & 100 & 71 & 83 \\
\hline $\begin{array}{l}\text { Total } \\
n=51\end{array}$ & 15 & 17 & 71 & 83 & 86 & 100 \\
\hline
\end{tabular}

Fuente: Historias Clínicas de Implantología oral.

Se registró un 9.8\% de complicaciones del total de las cirugías realizadas (Tabla 8). De ellas el $100 \%$ correspondieron a la técnica ventana lateral; no registrándose ninguna complicación tanto intra como post-operatoria en la técnica transcrestal. La perforación de la membrana de Scheneider durante el levantamiento constituyó la de más peso para un 5.8\% del total de complicaciones y precisó su oclusión con una membrana reabsorbible de colágeno. Solamente un $1.9 \%$ se correspondió con la presencia de septum óseos dentro del seno. Como complicaciones post-operatorias ocurrió un caso con epístaxis representando un $1.9 \%$ del total de complicaciones. Estos resultados son similares a los obtenidos por (Jiménez, y otros, 2015) reportando un 4.9\% de complicaciones todas asociadas a la técnica ventana lateral y en pacientes con gran reabsorción alveolar; demostró que la elevación del seno maxilar constituye una técnica quirúrgica segura en la gran mayoría de los pacientes intervenidos y que la incidencia de complicaciones intra y postoperatorias no afecta los resultados clínicos, sin embargo se puede asociar a un menor número de complicaciones la técnica indirecta, especialmente cuando esta va de la mano de la instalación de implantes pequeños. Con respecto a la técnica ventana lateral coincidimos con autores, (Avilés Echeverría, Hermida Salazar, \& Pineda Álvarez, 2020), (Bensaha \& Mjabber, 2016), (Quispe-Damián, Castro-Ruiz, \& Mendoza-Azpur, 2020), donde la perforación de la membrana es la complicación más frecuente, la principal causa es por un manejo inadecuado al momento de desprender la membrana de Schneider siendo 
más común en la parte inferior y lateral de la ventana, descrita por (Misch, 1993) el mismo sugiere tratamientos a este tipo de complicaciones.

Recientemente se ha propuesto el uso de instrumentos oscilantes ultrasónicos para cirugía de hueso como una alternativa al uso de piezas de alta velocidad para la preparación de la osteotomía de ventana lateral. La incidencia de reportes de perforación de membrana con éstos instrumentos varía del 3.6\% al 20\%. (Quispe-Damián, Castro-Ruiz, \& Mendoza-Azpur, 2020).

(Guzman Cruz \& Sauri Esquivel, 2017) reporta que las tasas de éxito en el abordaje lateral con o sin colocación de implantes son ciertamente tranquilizadoras, la perforación de la membrana sinusal es una complicación frecuente en este procedimiento, con tasas que van en un rango del $11 \%$ al $54 \%$. Teniendo en cuenta estas estadísticas podemos decir que los resultados de nuestra investigación son alentadores, ya que obtuvimos un $9.8 \%$ lo que está por debajo de ese rango.

Es importante destacar que factores como laceración de la membrana y el intento de repararla, aumenta considerablemente el tiempo del procedimiento y consecuentemente el malestar postoperatorio, sangramiento u otras variables intra-operatorias no fueron asociadas a la sinusitis post-operatoria; a diferencia de (Jiménez, y otros, 2015), el cual obtuvo una prevalencia del $4 \%$, asociado principalmente a la presencia de sinusitis previa y a la membrana sinusal delgada; la mayoría de los casos se observaron en mujeres, aunque no se reveló asociación estadística con el sexo del paciente.

Tabla 8: Complicaciones de la elevación del piso del seno maxilar según técnicas quirúrgicas.

\begin{tabular}{|c|c|c|c|c|c|c|c|}
\hline \multicolumn{2}{|c|}{ Complicaciones } & \multicolumn{6}{|c|}{ Técnicas Quirúrgicas } \\
\hline & & $\begin{array}{c}\text { Transcrestal } \\
n=15\end{array}$ & $\%$ & $\begin{array}{c}\text { Ventana } \\
\text { Lateral } \\
\mathrm{n}=36\end{array}$ & $\%$ & $\begin{array}{l}\text { Total } \\
\mathrm{n}=51\end{array}$ & $\%$ \\
\hline \multirow[t]{3}{*}{$\begin{array}{l}\text { Intra- } \\
\text { Operatorias }\end{array}$} & $\begin{array}{l}\text { Perforación o rotura } \\
\text { de la membrana de } \\
\text { Schneider durante el } \\
\text { levantamiento }\end{array}$ & - & - & 3 & 5.8 & 3 & 5.8 \\
\hline & $\begin{array}{l}\text { Presencia de septum } \\
\text { óseos dentro del } \\
\text { seno. }\end{array}$ & - & - & 1 & 1.9 & 1 & 1.9 \\
\hline & Hemorragias & - & - & - & - & - & - \\
\hline \multirow[t]{3}{*}{$\begin{array}{l}\text { Post- } \\
\text { Operatorias: }\end{array}$} & $\begin{array}{l}\text { Sangrado por la nariz } \\
\text { (Epístaxis) }\end{array}$ & - & - & 1 & 1.9 & 1 & 1.9 \\
\hline & $\begin{array}{l}\text { Despegamiento de la } \\
\text { herida }\end{array}$ & - & - & - & - & - & - \\
\hline & Sinusitis aguda & - & - & - & - & - & - \\
\hline
\end{tabular}


Presencia de fístula -

subantral

Infecciones.

Desplazamiento

implante al seno.
(

$-$

-

al General Complicaciones

Fuente: Historias Clínicas de Implantología oral.

\section{Conclusiones:}

- La elevación sinusal predominó en las edades de 41-50 años.

- Los dientes más relacionados fueron los premolares. Más de la mitad con gran déficit óseo.

- Se realizó ventana lateral en los casos con altura crestal menor de $8 \mathrm{~mm}$ con colocación del implante en una segunda fase. La técnica transcrestal se realizó a partir de 8-10 mm con la colocación simultánea del implante.

- La técnica más usada fue la ventana lateral y de localización unilateral.

- La complicación más frecuente fue la perforación de la membrana de Schneider en la técnica ventana lateral.

\section{Referencias bibliográficas}

Alsabbagh, A., Alsabbagh, M., Nahas, B., \& Rajih, S. (2017). Comparison of three different methods of internal sinus lifting for elevation heights of $7 \mathrm{~mm}$ an ex vivo study. Int J Implant Dent, Vol.3(40) pp 4-12.

Avila-ortiz, G., Wang, H., Galindo-moreno, P., Misch, C., \& Rudek, I. (2012). Neiva DDSR. Formation Following Maxillary Sinus Augmentation., Vol. 27(5).

Avilés Echeverría, S. A., Hermida Salazar, P. A., \& Pineda Álvarez, D. M. (2020). Elevación de seno maxilar con técnica de ventana lateral y rehabilitación implantoprotésica: reporte de caso. Research, Society and Development., Vol. 9 n.12.

Bensaha, T., \& Mjabber, H. (2016). Evaluation of new bone formation after sinus augmentation with two different methods. Int J Oral Maxillofac Surg., Vol. 45(1) pp 93-8.

Boyne, P., \& James, R. (1980). Grafting of the maxillary sinus floor with autogenous marrow and bone. J Oral Surg., Vol. 38 pp 613-6.

Bustillo, D. (2017). Elevación de piso de seno maxilar con técnica de ventana lateral y colocación simultánea de implantes: reporte de un caso. Clin. Periodoncia Implantol. Rehabil. Oral, Vol.10(3). 
C.D Aguirre Valencia, M. C. (2019). Técnica Transalveolar para el Levantamiento de Seno de Maxilares Atróficos. Trabajo Académico para optar el Título de Segunda Especialidad en: PERIODONCIA E IMPLANTOLOGIA, TACNA-PERÚ.

Cosci, F., \& Luccioli, M. (2000). A new sinus lifts technique in conjunction with placement of 265 implants: a 6-year retrospective study. Implant Dent., Vol. 9(4): pp 363-8.

Deporter, D., Todescan, R., \& Caudry, S. (2017). Simplifying management of the posterior maxilla using short, porous-surface dental implants and simultaneous indirect sinus elevation. Int J Periodontics Restorative Dent., Vol. 20 pp 476.

Duan, D., Fu, J., Qi, W., Du, Y., Pan, J., \& Wang, H. (2017). Graft-free maxillary sinus floor elevation: a systematic review and meta-analysis. J Periodontol., Vol. $88 \mathrm{pp}$ 550-64.

Esposito, M., Grusovin, M., Rees, J., Karasoulos, D., Felice, P., Alissa, R., \& al., e. (2010). Effectiveness of sinus lift procedures for dental implant rehabilitation: a Cochrane systematic review. Eur J Oral Implantol., Vol. 3(1): pp 7-26.

Guzman Cruz, D., \& Sauri Esquivel, E. (2017). Técnica de ventana lateral para elevación de seno maxilar con fines terapéuticos implantológicos. Revista Odontológica Latinoamericana. , Vol. 9(2): pp 69-74.

Herrmann, I., Lekholm, U., Holm, S., \& Kultje, C. (2005). Evaluation of patient and implant characteristics as potential prognostic factors for oral implant failures. Int J Oral Maxillofac Implants., Mar-Apr; Vol. 20(2): pp 220-30.

Jiménez, A., Monsalve, L., Ortiz, I., España, A., Segura, J., \& Velasco, E. (2015). La elevación del seno maxilar en el tratamiento con implantes dentales. Un estudio a 4 años. . Av Periodon Impl Oral., Vol. 27 pp 145-54.

Lambert, F., Leonard, A., Drion, P., Sourice, S., Layrolle, P., \& Rompen, E. (2017). Influence of space-fí- lling materials in subantral bone augmentation: Blood clot vs. autogenous bone chips vs. bovine hydroxyapatite. . Clin Oral Implants Res. , Vol. 22 pp 538-45.

Melej, C., Tirreau, B., \& Valdés, R. (2016). Elevación bilateral de seno maxilar con colocación inmediata y mediata de implantes. Rev ChilPeriodonOseoint. , Vol. 2(2) pp 3-10.

Misch, C. (1993). Implantología Contemporánea. Madrid: Mosby-Doyma Libros.

Nasser Nasser, K. (2020). La elevación indirecta del seno maxilar en el tratamiento con implates, técnica MISE. Tesis en opción al Grado de Doctor en Ciencias de la Salud. Universiad de Sevilla, España.

Nasser Nasser, K. e. (2018). El tratamiento con implantes mediante la elevación transcrestal del seno maxilar. Un estudio a 3 años. Avances en Odontoestomatología, Vol. 34 pp 151-58. 
Quispe-Damián, D., Castro-Ruiz, C., \& Mendoza-Azpur, G. (2020). Complicaciones quirúrgicas de la elevación de seno maxilar en Implantología. Odovtos International Journal of Dental Sciences, Vol. 22 pp 61-70.

Shalu, B., Ramesh, R., \& Komal, M. (2018). Aumento del seno maxilar. Indian Society Periodontology., Vol. 22(6) pp 468-73.

Summers, R. (1994). A new concept in maxillary implant surgery: the osteotome technique. Compendium: 15:152-162.

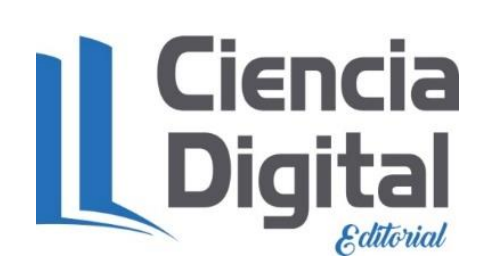




\section{PARA CITAR EL ARTÍCULO INDEXADO.}

Rodríguez Herrera, D., Lima Reyna, M. T., \& Pérez Iglesias, R. (2021). Elevación sinusal en pacientes con atrofia alveolar en el tratamiento de Implantología. Anatomía Digital, 4(3), 59-75. https://doi.org/10.33262/anatomiadigital.v4i3.1756

\section{Ciencia \\ LDigital}

El artículo que se publica es de exclusiva responsabilidad de los autores y no necesariamente reflejan el pensamiento de la Revista Anatomía Digital.

El artículo queda en propiedad de la revista y, por tanto, su publicación parcial y/o total en otro medio tiene que ser autorizado por el director de la Revista Anatomía Digital.
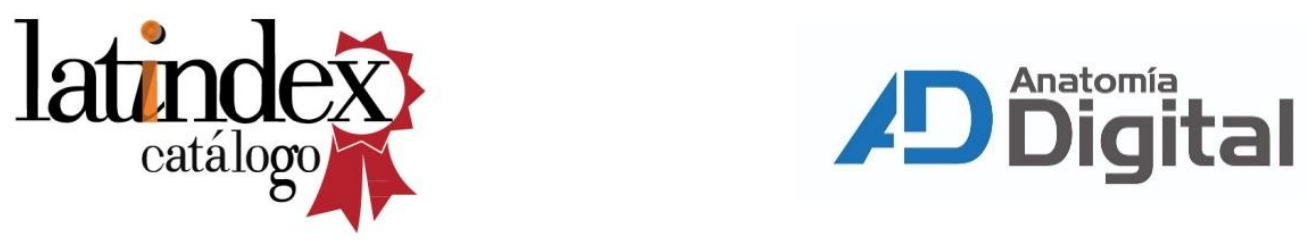